\title{
c-MET/VEGFR-2 co-localisation impacts on survival following bevacizumab therapy in epithelial ovarian cancer: an exploratory biomarker study of the phase 3 ICON7 trial
}

Robert D. Morgan ${ }^{1,2+} \oplus$, Cristina Ferreras ${ }^{1 \dagger}$, Isabel Peset ${ }^{3}$, Egle Avizienyte ${ }^{4}$, Andrew G. Renehan ${ }^{1,2}$, Richard J. Edmondson 1,5, Alexander D. Murphy², Shibani Nicum ${ }^{6}$, Thomas Van Brussel ${ }^{7}$, Andrew R. Clamp ${ }^{1,2}$, Diether Lambrechts ${ }^{7}$, Cong Zhou ${ }^{8+}$ and Gordon C. Jayson ${ }^{1,2^{*}+}$

\begin{abstract}
Introduction: Bevacizumab improves survival outcomes in women diagnosed with epithelial ovarian cancer (EOC). Pre-clinical data showed that the c-MET/NEGFR-2 heterocomplex negates VEGF inhibition through activation of c-MET signalling, leading to a more invasive and metastatic phenotype. We evaluated the clinical significance of c-MET and VEGFR-2 co-localisation and its association with VEGF pathway-related single nucleotide polymorphisms (SNPs) in women participating in the phase 3 trial, ICON7 (ClinicalTrials.gov identifier: NCT00262847).

Materials and methods: Patients had FIGO stage I-IIA grade 3/poorly differentiated or clear cell carcinoma or stage IIB-IV epithelial ovarian, primary peritoneal or fallopian tube cancer. Immunofluorescence staining for co-localised C-MET and VEGFR-2 on tissue microarrays and genotyping of germline DNA from peripheral blood leukocytes for VEGFA and VEGFR-2 SNPs was performed. The significance of these biomarkers was assessed against survival.

Results: Tissue microarrays from 178 women underwent immunofluorescence staining. Multivariable analysis showed that greater c-MET/NEGFR-2 co-localisation predicted worse OS in patients treated with bevacizumab after adjusting for FIGO stage and debulking surgery outcome (hazard ratio [HR] 1.034, 95\% confidence interval [95\% Cl] 1.010-1.059). Women in the c-MET/NEGFR-2 ${ }^{\mathrm{HIGH}}$ group treated with bevacizumab demonstrated significantly reduced OS (39.3 versus > 60 months; HR 2.00, 95\% Cl 1.08-3.72). Germline DNA from 449 women underwent genotyping. In the bevacizumab group, those women with the VEGFR-2 rs $2305945 \mathrm{G} / \mathrm{G}$ variant had a trend towards shorter PFS compared with $\mathrm{G} / \mathrm{T}$ or $\mathrm{T} / \mathrm{T}$ variants (18.3 versus 23.0 months; $\mathrm{HR} 0.74,95 \% \mathrm{Cl} 0.53-1.03$ ).
\end{abstract}

Conclusions: In bevacizumab-treated women diagnosed with EOC, high c-MET/NEGFR-2 co-localisation on tumour tissue and the VEGFR-2 rs 2305945 G/G variant, which may be biologically related, were associated with worse survival outcomes.

*Correspondence: Gordon.Jayson@nhs.net

${ }^{\dagger}$ Robert D. Morgan, Cristina Ferreras, Cong Zhou and Gordon C. Jayson

contributed equally to this work.

${ }^{2}$ The Christie NHS Foundation Trust, Manchester M20 4BX, UK

Full list of author information is available at the end of the article

(c) The Author(s) 2022. Open Access This article is licensed under a Creative Commons Attribution 4.0 International License, which permits use, sharing, adaptation, distribution and reproduction in any medium or format, as long as you give appropriate credit to the original author(s) and the source, provide a link to the Creative Commons licence, and indicate if changes were made. The images or other third party material in this article are included in the article's Creative Commons licence, unless indicated otherwise in a credit line to the material. If material is not included in the article's Creative Commons licence and your intended use is not permitted by statutory regulation or exceeds the permitted use, you will need to obtain permission directly from the copyright holder. To view a copy of this licence, visit http://creativecommons.org/licenses/by/4.0/. The Creative Commons Public Domain Dedication waiver (http://creativeco $\mathrm{mmons}$.org/publicdomain/zero/1.0/) applies to the data made available in this article, unless otherwise stated in a credit line to the data. 


\section{Highlights}

- Tissue microarrays and germline DNA from women recruited to the phase 3 trial, ICON7, underwent quantitative immunofluorescence for c-MET/VEGFR-2 co-expression and genetic sequencing for single nucleotide polymorphisms (SNPs) VEGF-pathway genes.

- High c-MET/VEGFR-2 co-localisation on tumour tissue independently predicted worse survival in bevacizumab-treated epithelial ovarian cancer.

- The VEGFR-2 rs2305945 SNPs also independently predict worse survival in bevacizumab-treated epithelial ovarian cancer.

Keywords: Epithelial ovarian cancer, Bevacizumab, c-MET, VEGFR-2, Single-nucleotide polymorphisms

\section{Introduction}

Ovarian cancer is the commonest cause of gynaecological cancer-related death in Europe and North America [1]. Multi-modality first-line therapy includes cytoreductive surgery plus platinum-based chemotherapy, followed by maintenance therapies in certain subgroups [2]. One such maintenance therapy is the anti-angiogenic agent, bevacizumab, a humanised monoclonal antibody directed against vascular endothelial growth factor (VEGF) [3]. Two randomised phase 3 trials, ICON7 and GOG-0218, demonstrated significant improvements in progression-free survival (PFS) using bevacizumab as part of the first-line therapy, with improvements in overall survival (OS) in patients at the highest risk of relapse [4-7]. Although clinical and pathological features can be used to guide patient selection for first-line bevacizumab therapy, no molecularly defined predictive biomarkers have been validated. While we have identified plasma Tie2 as the first response biomarker for VEGF inhibitors, we were unable to identify a biomarker that would predict the benefit from bevacizumab [8-10].

In our initial study of pre-treatment biomarkers, a group of patients were identified who appeared to be disadvantaged by bevacizumab $[8,9]$. The question is how such an effect can be induced by bevacizumab. A number of mechanisms have been described that account for tumour resistance to anti-angiogenic agents [11]. One involves the formation of a c-MET/VEGFR-2 heterocomplex [12]. VEGFR-2 and c-MET are receptor tyrosine kinases (RTKs) [13, 14]. VEGFR-2 dimerises the following binding to VEGF, and downstream RTK-mediated signalling leads to angiogenesis [13]. c-MET homodimerisation occurs following the binding of the hepatocyte growth factor (HGF) ligand, and RTK-mediated signalling leads to tumour proliferation and metastasis [14]. In a pre-clinical model of glioblastoma multiforme, $\mathrm{Lu}$ et al. demonstrated that following c-MET/VEGFR-2 heterocomplex formation, VEGF inhibits HGF/c-MET signalling by recruiting protein tyrosine phosphatase
1B (PTP1B) [12]. Thus, inhibition of VEGF using, for example, bevacizumab, negates the inhibitory effect of the c-MET/VEGFR-2 heterocomplex, thereby supporting $\mathrm{HGF} / \mathrm{c}-\mathrm{MET}$ signalling and driving tumorigenesis and progression [12]. To test the hypothesis that c-MET/ VEGFR-2 heterocomplex formation is responsible for patients experiencing a worse outcome from bevacizumab, we tested the clinical significance of c-MET/ VEGFR-2 co-localisation in epithelial ovarian cancer $(\mathrm{EOC})$ and refined the analysis further by genotyping germline single nucleotide polymorphisms (SNPs) in VEGF pathway-related genes to assess whether specific SNPs were associated with c-MET/VEGFR-2 co-localisation and survival outcomes.

\section{Methodology \\ Study participants}

All participants provided written informed consent for the International Collaboration of Ovarian Neoplasms 7 (ICON7) trial (ClinicalTrials.gov number, NCT00262847): an international, randomised, multicentre, open-label, phase 3 trial investigating the use of bevacizumab maintenance therapy as part of the firstline therapy in women diagnosed with high-risk FIGO (1988) stage I-IIA (grade 3/poorly differentiated tumours or clear cell carcinoma) or FIGO (1988) IIB-IV epithelial ovarian, primary peritoneal or fallopian tube cancer [5]. Progression-free survival (PFS) was defined as the time interval from the date of randomisation to the date of disease progression (defined clinically or radiologically, but not using CA125 criteria) or death. Overall survival (OS) was defined as the time interval from the date of randomisation to the date of death from any cause. All demographic and survival data were provided by the clinical trial centre co-ordinating ICON7 (Medical Research Council Clinical Trials Unit, University College London). As part of written informed consent, ICON7 participants could optionally agree to donate whole blood samples and pre-chemotherapy tumour tissue for translational 
research. Data presented here is from all patients who agreed to donate tumour tissue and/or whole blood samples. No case selection has been carried out.

\section{Immunofluorescence biomarkers}

Tissue microarrays (TMAs) from EOC tumour tissue sections underwent immunofluorescent staining using the BenchMark ULTRA system according to the RUO DISCOVERY universal staining procedure (Ventana Medical Systems). TMA sections were deparaffinised at $69^{\circ} \mathrm{C}$ for 8 min per cycle. Antigen retrieval occurred by pre-treatment with Cell Conditioning Solution 2 (CC2) (Ventana Medical Systems) and incubation at $95^{\circ} \mathrm{C}$ for 4 , $8,16,24$ and 32 min. TMA sections were then blocked by incubating with DISCOVERY inhibitor (Ventana Medical Systems) for $8 \mathrm{~min}$. Sections were warmed to $37^{\circ} \mathrm{C}$ and then incubated with the following primary antibodies: VEGF receptor 2 (55B11) rabbit monoclonal antibody (dilution 1:600; Cell Signalling Technology) and c-MET (1G7NB) mouse monoclonal antibody (dilution 1:300; Novus Biologicals) for $60 \mathrm{~min}$. Sections were then incubated with the corresponding HRP-conjugated secondary antibodies for $8 \mathrm{~min}$. The Tyramide Signal Amplification (PerkinElmer) was used on all slides to improve visualisation following incubation with the secondary antibody. Tyramide labelled with Cy3 (to visualise VEGFR-2, dilution 1:50; PerkinElmer) or FITC (to visualise c-MET, dilution 1:50; PerkinElmer) diluted in 1X Plus Amplification Diluent (PerkinElmer) were used.

Following the first staining, the second staining on the same section was performed using anti-human collagen type IV (dilution 1:300; Merck Millipore) to identify the stroma in each core. Sections were pre-treated with $\mathrm{CC} 2$ at $95^{\circ} \mathrm{C}$ for $4 \mathrm{~min}$ and then $8 \mathrm{~min}$. Slides were then cooled to $37^{\circ} \mathrm{C}$ before the second primary antibody was applied manually, followed by incubation for $60 \mathrm{~min}$. Slides were then incubated with a corresponding HRP-conjugated secondary antibody for 16 min followed by incubation with the Cy5 fluorophore-conjugated tyramide (dilution 1:50) diluted in $1 \mathrm{X}$ Plus Amplification Diluent for $8 \mathrm{~min}$. Slides were removed from the instrument and washed twice in EZ Prep buffer (Ventana Medical Systems). SlowFade Gold Antifade Mountant with 4',6-diamidino-2-phenylindole (DAPI) was added, and slides were mounted with coverslips.

Leica Aperio VERSA-200 digital pathology scanner was used to visualise immunofluorescence staining. Images were acquired at $\times 20$ magnification using the Andor iXon 888 camera (Additional File 1: Fig. S1). Immunofluorescence images from TMA sections were analysed by molecular pathology image analysis using Definiens Tissue Studio 2.7. Each TMA core was segmented into tumour and stroma by machine learning using collagen
IV staining to identify stroma. VEGFR-2 staining was used to detect vessels, and to calculate number of vessels, vessels density (number of vessels per tumour area), median vessel size and VEGFR-2 expression (mean of stain intensity) in the tumour component of each TMA core. c-MET/VEGFR-2 co-localisation was quantified using c-MET total intensity in VEGFR-2 positive vessel. The co-localisation values were not standardised by tumour area, vessel number and sizes because this information was included separately in data analysis.

\section{Genotyping biomarkers}

Peripheral blood samples were collected in K2EDTA blood tubes. Germline DNA was extracted from the precipitated leukocyte cell fraction. Genotyping was performed at the VIB Center for Cancer Biology (Leuven, Belgium) using the Sequenom MassARRAY ${ }^{\circledR}$ system (iPLEX GOLD) as reported previously [15]. Single nucleotide polymorphisms (SNPs) in VEGF-A and VEGFR-2 were analysed. A detailed description of the way in which SNPs were selected in these genes has been published previously $[15,16]$.

\section{Statistical power consideration}

In the ICON7 trial, bevacizumab maintenance therapy showed an PFS advantage of hazard ratio (HR) $0.81 \mathrm{com}$ pared to placebo [5]. A group of patients were however identified whom appeared to be disadvantaged by bevacizumab $[8,9]$. The HR between those patients whom benefitted versus those that did not was assumed to be 0.56 , a $30 \%$ reduction from the original HR of 0.81 . Based on a two-sided Cox proportional hazard regression analysis, it was estimated that a total number of 124 patients will be needed to achieve a significance level of 0.05 and a power of 0.8 , assuming data censorship was less than $25 \%$. Including a $15 \%$ contingency cohort, a minimum patient size of 143 would therefore be required. In total, samples from all 178 patients who gave consent to donate tumour tissue were employed in this study.

\section{Statistical analysis}

Variables from three types of data were analysed in this study: (1) clinical data, (2) immunofluorescence biomarker data including VEGFR-2 and c-MET expression and (3) genotyping biomarker data for SNPs in VEGF-A and $V E G F R-2$. The analysis started from investigating the associations between immunofluorescence variables and treatment outcomes (data types 1 and 2), followed by identifying SNPs that were associated with selected immunofluorescence biomarkers in matched patients (data types 2 and 3 ) and finally exploring the association between the identified SNPs and treatment outcomes (data types 1 and 3 ). 
The relationship between variables in clinical, immunofluorescence and genotyping data were assessed using one-way ANOVA for continuous variables or chi-squared tests for categorical variables.

The pre-treatment prognostic and predictive significance of each variable were assessed by including the biomarker as a sole covariate (univariable) in a proportional hazards model for PFS or OS. Here, prognostic significance refers to whether there is a significant association between a biomarker and survival independent of treatment arms, while predictive significance refers to if the association between a biomarker and survival are significantly different depending on treatment arms. Variables were included in continuous form where possible and were subject to transformation, such as $\log _{2}$ or dichotomisation by median. Assumption of proportionality was verified based on Schoenfeld residuals [17]. Martingale residuals from each marker specific analysis was examined for evidence of nonlinearity in the biomarker-hazard relationship [18]. Candidate biomarkers with $P$-values $\leq$ 0.05 in the univariable analysis were selected for subsequent multivariable proportional hazard regression analysis. A backward stepwise method was applied to identify the optimum subset of variables that associated with PFS or OS. Interactions between prognostic biomarkers of interest and treatment arms were included to seek predictive biomarkers that predicted benefit from bevacizumab. To address potential confounding factors, clinical variables that are significantly associated with immunofluorescence biomarkers are included in multivariable models regardless of their association with patient survival. Their interaction with immunofluorescence biomarkers were also interrogated.

Genotyping data from VEGF-A and VEGFR-2 were introduced to explore potential biological mechanisms underpinning the immunofluorescence data. The SNPs were filtered by their association with immunofluorescence biomarkers and those that strongly associated were investigated for their prognostic and predictive significance with respect to PFS/OS using the proportional hazard model described above. Analyses were carried out in accordance with the REMARK guidelines [19] and were implemented using R 3.6.0 [20].

\section{Results}

\section{Immunofluorescence biomarkers}

To test the clinical significance of c-MET/VEGFR-2 colocalisation in samples from 178 patients diagnosed with EOC, a TMA-based immunofluorescence staining method was developed for c-MET and VEGFR-2. The statistics of key immunofluorescence biomarkers are summarised in Additional File 2: Table S1. The clinical data from these 178 cases was similar to the original
ICON7 population with a slightly higher proportion of clear cell carcinoma cases in the immunofluorescence biomarker study, although the difference was not significant (Fisher's exact test, $P=0.07$ ) (Table 1 ). In this group of 178 patients, those with FIGO stage III/IV disease demonstrated significantly higher vessel density $(P$ $=0.005)$, number of vessels $(P=0.004)$, median vessel size $(P=0.006)$ and VEGFR-2 expression $(P=0.003)$; consistent with the concept that angiogenesis is associated with tumour growth and metastasis (Additional File 2: Table S1).

The association between vascular tissue markers of angiogenesis and higher FIGO stage provided confidence in the validity of the immunofluorescence biomarkers and sample set, supporting further evaluation of the relationship between immunofluorescence biomarkers and survival outcomes. As a first step, a univariable analysis of clinical prognostic factors and vascular tissue biomarkers was performed (Additional File 2: Table S2). Both FIGO stage (III/IV vs. I/II) and amount of residual disease $(\leq 1$ $\mathrm{cm}$ vs. $>1 \mathrm{~cm}$ ) following debulking surgery were of prognostic significance, in keeping with other ovarian cancer datasets (Additional File 2: Table S2) $[6,7]$. In addition, number of vessels $(P=0.015)$ and VEGFR-2 expression $(P=0.027)$ inversely associated with PFS regardless of treatment (Additional File 2: Table S2). Importantly, in this univariable analysis, c-MET/VEGFR-2 co-localisation predicted significantly worse $\operatorname{OS}(P=0.001)$ in patients treated with bevacizumab (Additional File 2: Table S2).

We next carried out a multivariable analysis to interrogate whether immunofluorescence biomarkers could predict treatment benefit of bevacizumab, but not placebo, with established prognostic clinical factors. Consistent with other ovarian cancer studies, FIGO stage (III/IV vs. I/II; hazard ratio [HR] 2.384, 95\% confidence interval [95\%CI] 1.181-4.815, $P=0.015$ ) and amount of residual disease ( $\leq 1 \mathrm{~cm}$ vs. $>1 \mathrm{~cm}$; HR 0.243 , 95\%CI 0.147-0.399, $P<0.001)$ were significant prognostic factors for PFS (Table 2). For OS, the amount of residual disease was also significant ( $\leq 1 \mathrm{~cm}$ vs. $>1 \mathrm{~cm}$; HR 0.397 , 95\%CI $0.228-$ $0.694, P=0.001$; Table 2). Multivariable analysis showed that greater c-MET/VEGFR-2 co-localisation predicted worse $O S$ in patients treated with bevacizumab after adjusting for FIGO stage and debulking surgery outcome (HR 1.034, 95\%CI 1.010-1.059, $P=0.006$ ) (Table 2). Furthermore, by dichotomising patients according to the median expression of c-MET/VEGFR-2 into c-MET/ VEGFR-2 ${ }^{\text {LOW }}(\leq$ median$)$ and c-MET/VEGFR-2 ${ }^{\text {HIGH }}(>$ median) groups, data showed that patients with c-MET/ VEGFR-2 ${ }^{\mathrm{HIGH}}$ tumours treated with bevacizumab had significantly reduced OS compared to c-MET/VEGFR$2^{\text {LOW }}$ tumours (39.3 vs. > 60 months; multivariable HR 
Table 1 Demographic data. Data is presented as median (range) or number (percentage). C carboplatin, $P$ paclitaxel, B bevacizumab

\begin{tabular}{|c|c|c|c|c|c|c|}
\hline \multirow[t]{2}{*}{ Clinical data } & \multicolumn{2}{|c|}{$\begin{array}{l}\text { Immunofluorescence biomarker } \\
\text { study }(n=178)\end{array}$} & \multicolumn{2}{|c|}{$\begin{array}{l}\text { Genotyping biomarker study ( } N= \\
\text { 449) }\end{array}$} & \multicolumn{2}{|c|}{ ICON7 population $(N=1528)$} \\
\hline & $\mathrm{C}+\mathrm{P}(N=91)$ & $\mathrm{C}+\mathrm{P}+\mathrm{B}(N=87)$ & $\mathrm{C}+\mathrm{P}(N=216)$ & $\mathrm{C}+\mathrm{P}+\mathrm{B}(N=233)$ & $\mathrm{C}+\mathrm{P}(N=764)$ & $\mathrm{C}+\mathrm{P}+\mathrm{B}(N=764)$ \\
\hline Age, years & $58(35-76)$ & $56(25-75)$ & $58(24-79)$ & $56(24-77)$ & $57(18-81)$ & $57(24-82)$ \\
\hline \multicolumn{7}{|l|}{ ECOG performance status } \\
\hline 0 & $32(35)$ & $38(44)$ & $87(40)$ & $105(45)$ & $358(47)$ & $334(44)$ \\
\hline 1 & $52(57)$ & $45(52)$ & $111(51)$ & $117(50)$ & $354(47)$ & $366(48)$ \\
\hline 2 & $2(2)$ & $2(2)$ & $13(6)$ & $7(3)$ & $43(6)$ & $45(6)$ \\
\hline Unknown & $5(5)$ & $2(2)$ & $5(2)$ & $4(2)$ & $9(1)$ & $19(2)$ \\
\hline \multicolumn{7}{|l|}{ Histological subtype } \\
\hline Serous & $61(67)$ & $56(64)$ & $148(69)$ & $158(68)$ & $529(69)$ & $525(69)$ \\
\hline Mucinous & 0 & $1(1)$ & $3(1)$ & $2(1)$ & $15(2)$ & $19(2)$ \\
\hline Endometrioid & $8(9)$ & $4(5)$ & $17(8)$ & $15(6)$ & $57(7)$ & $60(8)$ \\
\hline Clear cell & $14(15)$ & $15(16)$ & $23(11)$ & $30(13)$ & $60(8)$ & $67(9)$ \\
\hline Mixed & $5(5)$ & $10(11)$ & $13(6)$ & $21(9)$ & $48(6)$ & $50(5)$ \\
\hline Others & $3(3)$ & $1(1)$ & $12(6)$ & $7(3)$ & $55(7)$ & $53(7)$ \\
\hline \multicolumn{7}{|l|}{ Histological grade } \\
\hline 1 (well differentiated) & $2(2)$ & $2(2)$ & $10(5)$ & $12(5)$ & $57(7)$ & $41(5)$ \\
\hline 2 (moderately differentiated) & $13(14)$ & $11(13)$ & $34(16)$ & $39(17)$ & $142(19)$ & $175(23)$ \\
\hline 3 (poorly differentiated) & $76(84)$ & $73(84)$ & $169(78)$ & $179(77)$ & $556(74)$ & $538(71)$ \\
\hline Unknown & 0 & $1(1)$ & $3(1)$ & $3(1)$ & 10 & 10 \\
\hline \multicolumn{7}{|l|}{ FIGO (1988) stage } \\
\hline$|/| \mid$ & $17(19)$ & $22(25)$ & $46(21)$ & $44(19)$ & $145(19)$ & $137(18)$ \\
\hline III/IV & $74(81)$ & $65(75)$ & $170(79)$ & $189(81)$ & $619(81)$ & $627(82)$ \\
\hline \multicolumn{7}{|l|}{ Debulking surgery } \\
\hline$>1 \mathrm{~cm}$ residual disease & $35(38)$ & $26(30)$ & $62(29)$ & $61(26)$ & $195(26)$ & $192(26)$ \\
\hline$\leq 1 \mathrm{~cm}$ residual disease & $56(62)$ & $61(70)$ & $143(71)$ & $167(74)$ & $569(76)$ & $572(76)$ \\
\hline
\end{tabular}

Table 2 Multivariable survival analysis for immunofluorescence biomarkers. 95\% Cl 95\% confidence interval, HR hazard ratio, PFS progression-free survival, OS overall survival. In the multivariable analysis, a P-value cut-off of $\leq 0.05$ was considered statistically significant. Predictive model, a model exploring if the association between a biomarker and survival are significantly different depending on treatment arms: $\mathrm{HR}, 95 \% \mathrm{Cl}$ and P-values come from the interaction term. Clinical factors prognostic for PFS/OS were included in the model

\begin{tabular}{|c|c|c|c|c|c|c|}
\hline \multirow[t]{3}{*}{ Covariate name } & \multicolumn{6}{|c|}{ Predictive model } \\
\hline & \multicolumn{3}{|l|}{ PFS } & \multicolumn{3}{|l|}{ os } \\
\hline & HR & $95 \% \mathrm{Cl}$ & $P$-value & $\overline{H R}$ & $95 \% \mathrm{Cl}$ & $P$-value \\
\hline \multicolumn{7}{|l|}{ Clinical factors } \\
\hline FIGO stage (III/IV vs. I/II) & 2.384 & $1.181-4.815$ & 0.015 & 1.994 & $0.893-4.454$ & 0.092 \\
\hline Debulking surgery outcome ( $\leq 1 \mathrm{~cm}$ vs. $>1 \mathrm{~cm}$ residual disease) & 0.243 & $0.147-0.399$ & $<0.001$ & 0.397 & $0.227-0.695$ & 0.001 \\
\hline \multicolumn{7}{|l|}{ Immunofluorescence biomarker } \\
\hline c-MET/NEGFR-2 co-localisation (increase of expression) & 1.011 & $1.004-1.018$ & 0.003 & 1.001 & $0.995-1.008$ & 0.671 \\
\hline Bevacizumab arm & - & - & ns & 1.116 & $0.995-1.008$ & 0.669 \\
\hline \multicolumn{7}{|l|}{ Predictive significance } \\
\hline c-MET/NEGFR-2 co-localisation in bevacizumab arm (interaction) & - & - & ns & 1.034 & $1.010-1.059$ & 0.006 \\
\hline
\end{tabular}


2.00, 95\%CI 1.08-3.72, $P=0.03$; Fig. 1A) whereas in the placebo group, there was no significant difference (multivariable HR 1.26, 95\%CI 0.67-2.39, $P=0.47$; Fig. 1B).

\section{Genotyping biomarker}

Four hundred and forty-nine patients underwent SNP genotyping: 216 in the control group and 233 in the

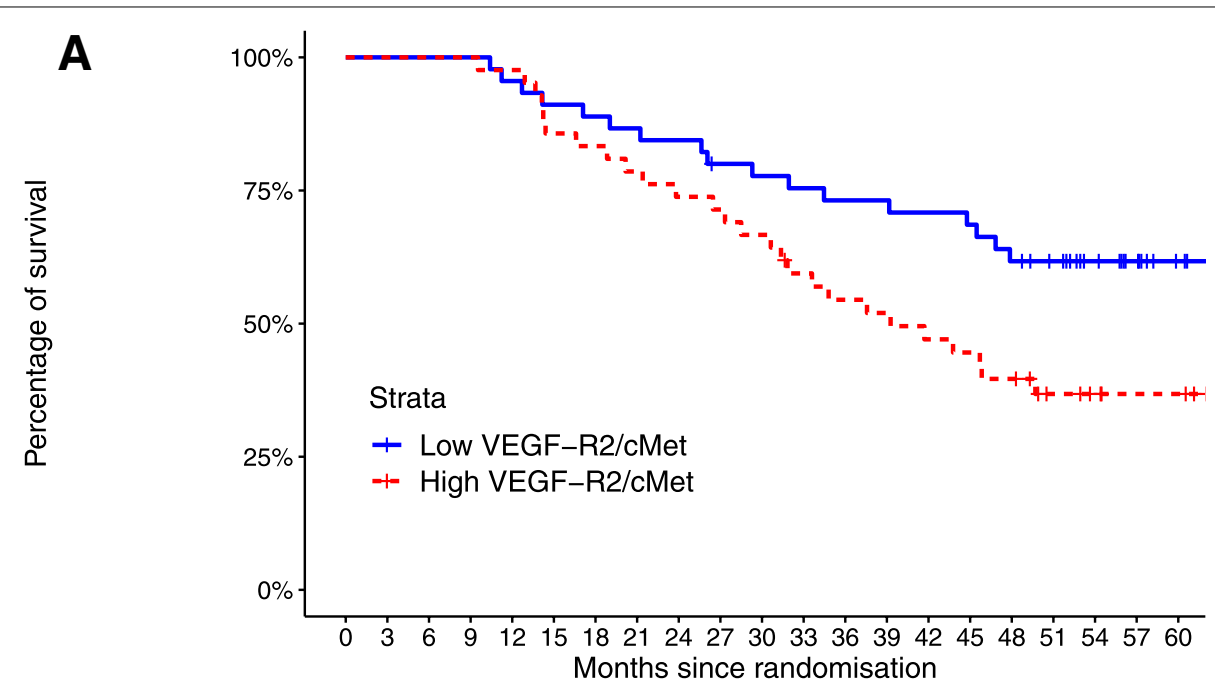

Number at risk

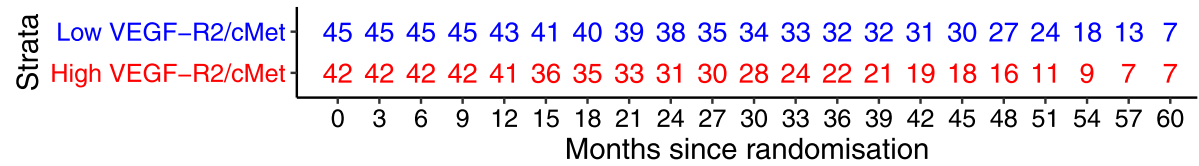

B
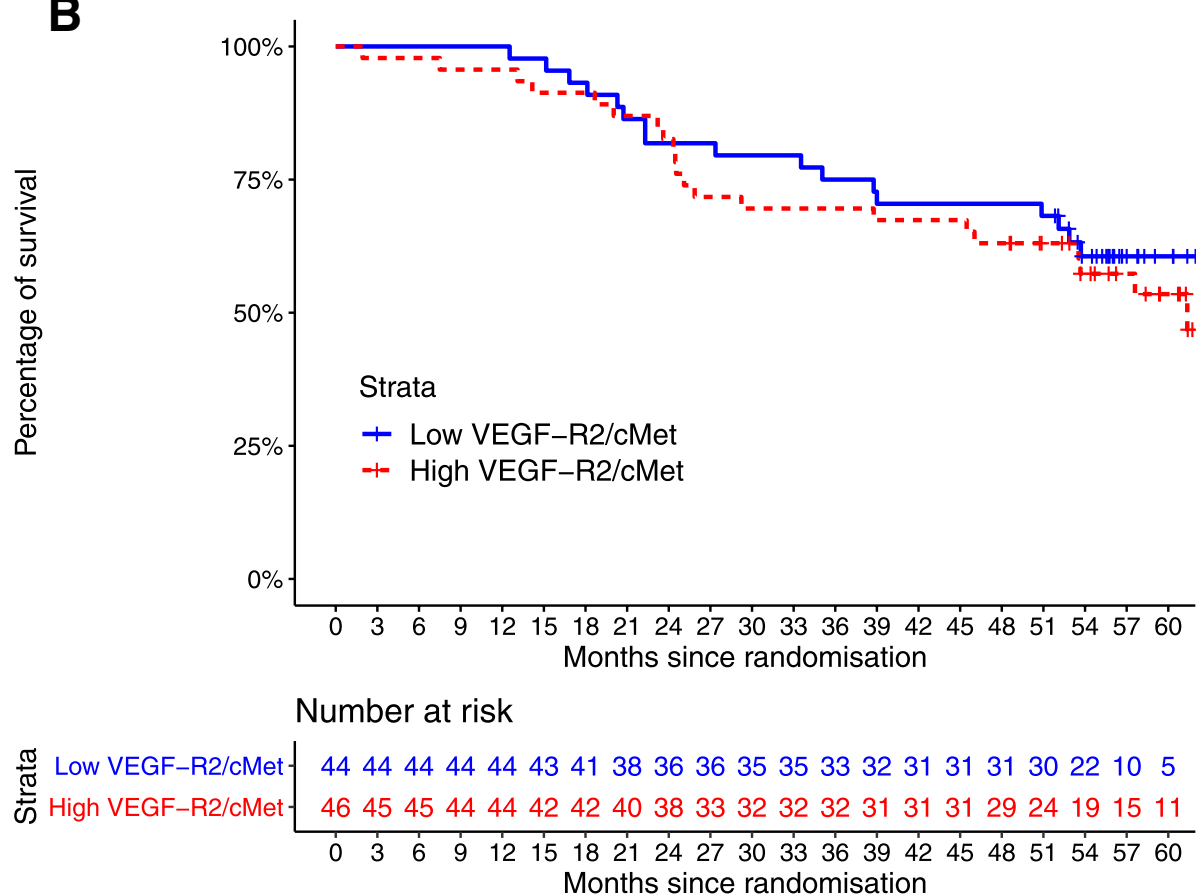

Fig. 1 A Kaplan-Meier estimates of overall survival in patients $(n=87)$ treated with carboplatin/paclitaxel plus bevacizumab (experimental group). Patients are dichotomised by their median average expression of c-METNEGFR-2 into c-MET/NEGFR-2 ${ }^{\text {HIGH }}(n=42)$ and c-MET/NEGFR-2 ${ }^{\text {LOW }}(n=45)$ groups. The median OS was 39.3 months for c-MET/NEGFR-2 ${ }^{\text {HIGH }}$ and $>60$ months c-MET/NEGFR-2 ${ }^{\text {LOW }}$ (HR 2.00, $P=0.03$ ). B Kaplan-Meier estimates of overall survival in patients $(n=90)$ treated with carboplatin/paclitaxel (control group). Patients are dichotomised by their median average expression of c-MET/NEGFR-2 into c-MET/NEGFR-2 ${ }^{\text {HIGH }}(n=46)$ and C-MET/NEGFR-2 LOW $(n=44)$ groups. Both groups had a median OS $>60$ months. 
bevacizumab group (Table 1). Thirty-five $V E G F-A$ and $V E G F R-2$ SNPs were investigated to understand the potential genetic mechanisms underpinning the variation in c-MET/VEGFR-2 co-localisation (Additional File 2: Table S3). Focusing on the 142 matched patients who underwent both immunofluorescence studies and genotyping, three SNPs were identified that were strongly associated with high co-localisation of c-MET and VEGFR-2. These included VEGF-A rs3025033 (G variant; chi-squared test, $P=0.138$ ), VEGFR-2 rs1870377 (A variant; chi-squared test, $P=0.151$ ) and VEGFR-2 rs2305945 (G variant; chi-squared test, $P=$ 0.047) (Additional File 2: Table S3). The prognostic and predictive significance of these SNPs were evaluated, with univariable and multivariable results presented in Additional File 2: Table S3 and Table 3, respectively. Of these three SNPs, VEGFR-2 rs2305945 independently predicted PFS in the bevacizumab-treated cohort after adjusting for known prognostic clinical factors (multivariable HR 1.592, 95\%CI 1.110-2.284, $P=0.012$ ) (Table 3). Patients with the VEGFR-2 rs2305945 G/G variant who were treated with bevacizumab had shorter PFS than those with G/T or T/T variants (18.3 vs. 23.0 months; HR 0.74, 95\%CI 0.53-1.03, $P=0.07$; Fig. 2A). In contrast, there was moderately longer PFS in patients with the VEGFR-2 rs2305945 G/G variant who were treated with chemotherapy alone (21.2 [G/G variant] vs. 15.6 months [G/T or T/T variants]; HR 1.14, $95 \% 0.79-1.63, P=0.48$; Fig. $2 \mathrm{~B})$. The frequency of each variant in the three key SNPs are shown in Additional File 2: Table S4. The Kaplan-Meier curves of PFS for VEGF-A rs3025033 and VEGFR-2 rs1870377 SNPs are shown in Additional File 2: Fig. S2.

\section{Immunofluorescence and genotyping biomarkers}

The two multivariable analyses show that c-MET/ VEGFR-2 co-localisation (178 patients) and VEGFR-2 rs2305945 (449 patients) are independent predictive factors for bevacizumab-associated outcomes, supporting the hypothesis that there are a group of patients whose outcome is adversely affected by treating with bevacizumab. Furthermore, the VEGFR-2 rs2305945 G/G variant is associated with increasing c-MET/VEGFR-2 co-localisation $(P=0.047$; Additional File 2: Table S3) and each biomarker predicts worse PFS (VEGFR2 rs2305945: HR 1.592, $P=0.012$; Table 3) and OS (c-MET/VEGFR-2 co-localisation: HR 1.034, $P=0.006$; Table 2) in patients treated with bevacizumab, suggesting a possible biological and/or clinical interaction (Additional File 1: Fig. S3). An exploratory analysis on the relationship between c-MET/VEGFR-2 co-localisation and the VEGFR-2 rs2305945 SNP was carried out by investigating their interaction in one multivariable model (Additional File 2: Table S5). It was observed that patients had worse PFS and OS if they had high c-MET/VEGFR-2 co-localisation and the VEGFR-2 rs $2305945 \mathrm{G} / \mathrm{G}$ variant, although the difference was not significant $(P=0.071$ for PFS and $P=0.272$ for OS; Additional File 2: Table S5).

\section{Discussion}

In our initial studies of biomarkers that predict benefit from bevacizumab in ovarian cancer, we identified a group of patients who appeared to be disadvantaged

Table 3 Multivariable survival analysis for genotype biomarkers. ${ }^{*} \mathrm{G}$ allele. 95\% Cl 95\% confidence interval, $H R$ hazard ratio, $R D$ residual disease, PFS progression-free survival, OS overall survival. In the multivariable analysis, a $P$-value cut-off of $\leq 0.05$ was considered statistically significant. Predictive model, a model exploring if the association between a biomarker and survival are significantly different depending on treatment arms: $\mathrm{HR}, 95 \% \mathrm{Cl}$ and $P$-values come from the interaction term. Clinical factors prognostic for PFS/OS were included in the model

\begin{tabular}{|c|c|c|c|c|c|c|}
\hline \multirow[t]{3}{*}{ Covariate name } & \multicolumn{6}{|c|}{ Predictive model } \\
\hline & \multicolumn{3}{|l|}{ PFS } & \multicolumn{3}{|l|}{ os } \\
\hline & HR & $95 \% \mathrm{Cl}$ & $P$-value & HR & $95 \% \mathrm{Cl}$ & $P$-value \\
\hline \multicolumn{7}{|l|}{ Clinical biomarker } \\
\hline FIGO stage (III/IV vs. I/II) & 3.171 & $2.118-4.747$ & $<0.001$ & 2.434 & $1.409-4.205$ & 0.001 \\
\hline $\begin{array}{l}\text { Debulking surgery outcome }(\leq 1 \\
\mathrm{cm} \text { vs. }>1 \mathrm{~cm} \mathrm{RD)}\end{array}$ & 0.506 & $0.394-0.651$ & $<0.001$ & 0.396 & $0.274-0.573$ & $<0.001$ \\
\hline \multicolumn{7}{|l|}{ Genotype biomarker } \\
\hline rs2305945 (VEGFR-2)* & 0.794 & $0.961-1.650$ & 0.095 & - & - & ns \\
\hline Bevacizumab & 0.497 & $0.296-0.833$ & 0.008 & - & - & ns \\
\hline \multicolumn{7}{|l|}{ Predictive significance } \\
\hline $\begin{array}{l}\text { rs2305945 (VEGFR-2) in the beva- } \\
\text { cizumab arm* (interaction) }\end{array}$ & 1.592 & $1.110-2.284$ & 0.012 & - & - & ns \\
\hline
\end{tabular}




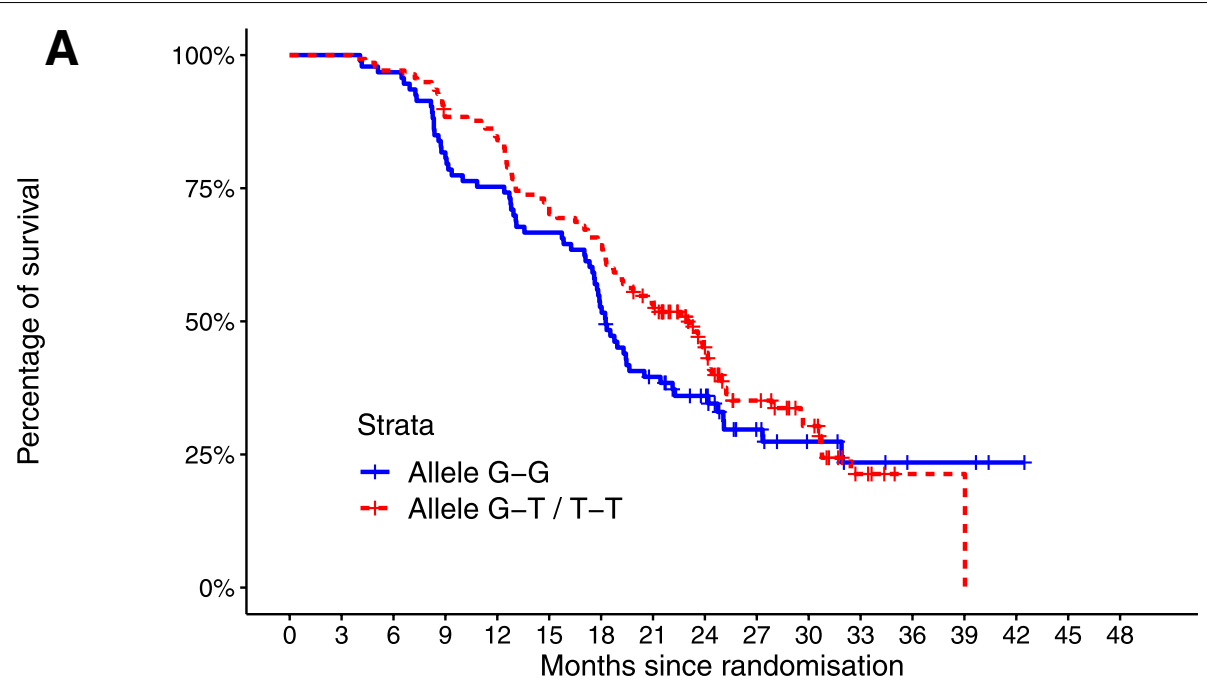

Number at risk

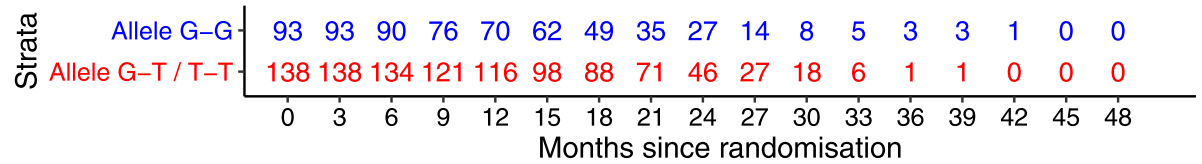

B

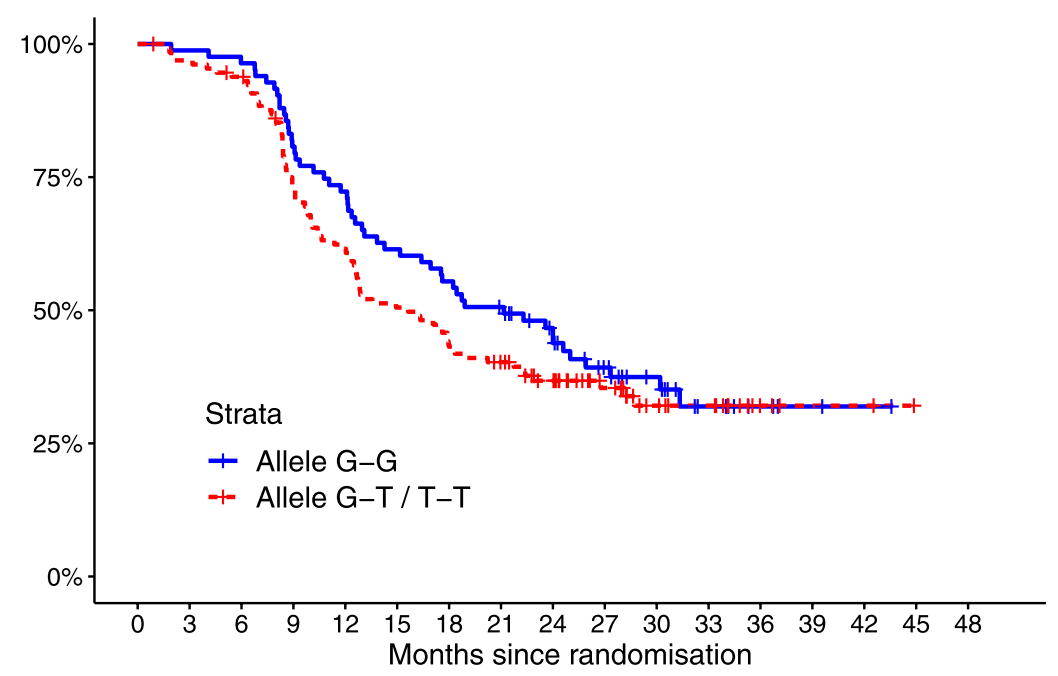

Number at risk

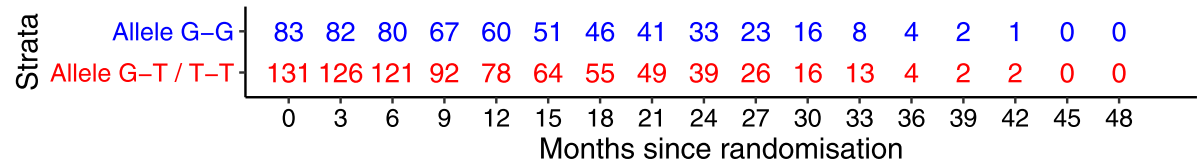

Fig. 2 A Kaplan-Meier estimates of progression-free survival in patients $(n=231)$ treated with carboplatin/paclitaxel plus bevacizumab (experimental group). Patients are separated into two groups: those with the VEGFR-2 rs $2305945 \mathrm{G} / \mathrm{G}$ variant $(n=93)$ and those with the VEGFR-2 rs2305945 G/T or T/T variant $(n=138)$. The median PFS was 18.2 months for those patients with the VEGFR-2 rs $2305945 \mathrm{G} / \mathrm{G}$ variant and 23.0 months for those with the VEGFR-2 rs2305945 G/T or T/T variant (HR 0.74, $P=0.07$ ). B Kaplan-Meier estimates of progression-free survival in patients $(n=214)$ treated with carboplatin/paclitaxel (control group). Patients are separated into two groups: those with the VEGFR-2 rs $2305945 \mathrm{G} / \mathrm{G}$ variant $(n=83)$ and those with the VEGFR-2 rs $2305945 \mathrm{G} / \mathrm{T}$ or T/T variant $(n=131)$. The median PFS was 21.2 months for those patients with the VEGFR-2 rs $2305945 \mathrm{G} / \mathrm{G}$ variant and 15.6 months for those with the VEGFR-2 rs2305945 G/T or T/T variant $(H R 1.14, P=0.48)$ 
by bevacizumab $[8,9]$. The question was what potential mechanism(s) could have been responsible for this observation? A contemporaneous publication had identified a mechanism in which c-MET and VEGFR-2 were fused in a heterocomplex and subsequent VEGF inhibitor-mediated inhibition of VEGFR-2 activated c-MET signalling by inhibition of the phosphatase, PTP1B [12]. Thus, we hypothesised that expression of the c-MET/VEGFR-2 heterocomplex might account for the adverse outcome of a subgroup of bevacizumab-treated patients. Here, by using immunofluorescence image analysis studies to detect co-localisation of c-MET and VEGFR-2 as a surrogate for heterocomplex expression, we have shown that co-localisation, particularly in the context of the VEGFR2 rs2305945 G/G variant, predicted a worse outcome following bevacizumab treatment. These two multivariable analyses show that c-MET/VEGFR-2 co-localisation and the VEGFR-2 rs2305945 G/G variant are independent predictive factors for bevacizumab-associated outcomes; supporting the hypothesis that there are a group of patients whose outcome is adversely affected through treatment with bevacizumab.

The immunofluorescence image analysis reported here provides the first clinical data from a prospective clinical trial supporting c-MET/VEGFR-2 heterocomplex formation as a putative resistance mechanism against VEGF inhibition [12]. The interesting finding that higher levels of c-MET/VEGFR-2 co-localisation were associated with worse OS provides evidence to support the use of alternative therapies for such patients; in particular, targeted agents that inhibit both c-MET and VEGFR-2 kinase domains.

The HGF/c-MET axis can be inhibited through small molecule tyrosine-kinase inhibitors or monoclonal antibodies directed against either c-MET or HGF. Two oral small-molecule multi-kinase inhibitors with activity against c-MET: cabozantinib [21] and crizotinib [22], are effective in renal, thyroid and lung cancer [23-25]. Cabozantinib is active against both c-MET and VEGFR-2 and could be considered in future trials recruiting patients diagnosed with EOC where their tumour manifests colocalisation of c-MET/VEGFR-2. Indeed, three phase II trials with cabozantinib have reported modest activity in chemo-resistant ovarian cancer, although none of the trials incorporated a biomarker [26-28].

We were interested in VEGF pathway-related SNPs that pertained to the c-MET/VEGFR-2 heterocomplex. The VEGFR-2 rs2305945 G/G variant was found to be significantly associated with c-MET/VEGFR-2 co-localisation and while the co-localisation predicted worse outcome from bevacizumab in OS, the SNP predicted worse outcome from bevacizumab in PFS. Worse outcomes from bevacizumab, though not significant, were observed in patients whose tumours manifested both c-MET/ VEGFR-2 co-localisation and VEGFR-2 rs2305945 G/G variant. However, it should be noted that this analysis was carried out in a small cohort of bevacizumab-treated patients with both immunofluorescence and genotyping data available and evaluation of the impact of the two factors on survival is confounded by the potential that the SNP could influence c-MET/VEGFR-2 heterocomplex formation.

Accepting that there is an association between VEGFR2 rs2305945 and c-MET/VEGFR-2 expression, the question is whether there is any evidence for a biological role of this SNP in the c-MET/VEGFR-2 heterocomplex? Although this SNP is located within a non-coding region of $V E G F R-2$, there are data suggesting it is of clinical interest. Indeed, previous data has reported that rs2305945 is associated with differential response to VEGF inhibitors in age-related macular degeneration and also protective against ovarian hyperstimulation syndrome $[29,30]$. The latter is of particular relevance here given the physiological importance of VEGF to follicular cysts formation and the plethora of trials that have demonstrated the clinical activity of VEGF inhibitors in ovarian cancer, irrespective of the platinum/progression-free interval.

The main strength of the clinical data reported in this study is that it was prospectively collected, although it represents only a proportion of the patients recruited to ICON7. In addition, genotyping biomarker assessment was performed using an established methodology [15, 16]. As an exploratory study for biomarker discovery, no correction was made for multiple testing in the analysis; instead, chance of false discovery was controlled by verifying against biological rationale. For example, only SNPs from the VEGF pathway were chosen for analysis because of their putative molecular relevance to bevacizumab and the VEGF-A/VEGFR-2 signalling pathway. These SNPs were filtered by their association with the immunofluorescence biomarkers before carrying out survival analysis. Further clinical studies will be needed to corroborate the findings, but confidence in the results can be gained from the similarity in behaviour of conventional prognostic factors with the main ICON7 study [5]. Moreover, this study and the translational work from GOG-0218 reported the potential predictive value of vessel density for bevacizumab-treated patients [31].

The main limitation of our study is the sample size, with $11.6 \%(178 / 1528)$ and $29.4 \%(449 / 1528)$ of women from ICON7 donating tumour tissue and/or blood, respectively. It is notable however, that donation of research tumour tissue and/or blood samples was optional, and not mandated within the trial protocol. Therefore, the sample size reflects the proportion of 
women willing to donate translational research samples. Moreover, the number required to detect differences in c-MET/VEGFR-2 co-localisation, according to the statistical power consideration, was achieved in the study. Another limitation of this biomarker study is the absence of an independent validation cohort and this could be assessed in an appropriately powered prospective study.

In conclusion, in bevacizumab-treated patients diagnosed with EOC, high c-MET/VEGFR-2 co-localisation on tumour tissue and the VEGFR-2 rs2305945 G/G variant, which may be biologically related, was associated with worse survival outcomes. In patients who did not receive bevacizumab, high c-MET/VEGFR-2 co-localisation showed no association with outcome.

\section{Supplementary Information}

The online version contains supplementary material available at https://doi. org/10.1186/s12916-022-02270-y.

Additional file 1: Figure S1- Examples of tissue microarray immunofluorescence staining for VEGFR-2 and c-MET. Figure S2- Progression-free survival for VEGF-A rs3025033 and VEGFR-2 rs 1870377 SNPs in the control (chemotherapy) and experimental (chemotherapy plus bevacizumab) group. Figure S3- Summary of findings for c-MET/VEGFR-2 co-localisation and SNP VEGFR-2 rs2305945.

Additional file 2: Table S1-Characteristics of immunofluorescence biomarkers and their association with demographic data. Table S2- Prognostic and predictive biomarkers assessed as significant in the univariable analysis. Table S3- List of the 35 SNPS in VEGF-A and VEGFR-2 tested and their association with c-MET/NEGFR-2 co-localisation. Table S4- Frequency of three key SNPs in VEGF-related genes. Table S5- Relationship between c-MET/NEGFR-2 co-localisation and VEGFR-2 rs2305945 SNP.

\section{Acknowledgements}

We acknowledge the contribution of the Cancer Research UK Cambridge Institute for the work involved in building the tissue microarray blocks.

\section{Authors' contributions}

Design and conception of study: CZ and GCJ. Data collection and assembly: RDM, CF, IP, DL, CZ and GCJ. Data analysis and interpretation: RDM, CF, IP, RJE, $A R C, D L, C Z$ and GCJ. Writing of the manuscript: RDM , CF, IP, RJE, ARC, DL, CZ and GCJ. All authors read and approved the final manuscript.

\section{Funding}

This work was supported by Wellbeing of Women (Award Ref RG1705).

\section{Availability of data and materials}

All information regarding patients is strictly confidential.

\section{Declarations}

\section{Ethics approval and consent to participate}

All women included in this study provided written informed consent to participate in the ICON7 trial (clinicaltrials.gov identifier: NCT00483782). The study was performed in accordance with the Declaration of Helsinki and adhered to the Good Clinical Practice guidelines.

\section{Competing interests}

The authors declare that they have no competing interests.

\section{Author details}

${ }^{1}$ Division of Cancer Sciences, Faculty of Biology, Health and Medicine, University of Manchester, Manchester, UK. ${ }^{2}$ The Christie NHS Foundation Trust, Manchester M20 4BX, UK. ${ }^{3}$ Medicines Discovery Catapult, Alderley Park, Cheshire, UK. ${ }^{4}$ Cancer Communications and Consultancy Ltd., Knutsford, UK. ${ }^{5}$ Manchester University NHS Foundation Trust, Manchester, UK. ${ }^{6}$ Oxford University Hospitals NHS Foundation Trust, Oxford, UK. ${ }^{7}$ Leuven Center for Cancer Biology, University of Leuven, Leuven, Belgium. ${ }^{8}$ Cancer Biomarker Centre, Cancer Research UK Manchester Institute, Manchester, UK.

Received: 7 October 2021 Accepted: 20 January 2022

Published online: 11 February 2022

\section{References}

1. Torre $L A$, Trabert B, DeSantis CE, Miller KD, Samimi G, Runowicz CD, et al. Ovarian cancer statistics, 2018. CA Cancer J Clin. 2018;68(4):284-96.

2. Jayson GC, Kohn EC, Kitchener HC, Ledermann JA. Ovarian cancer. Lancet. 2014;384(9951):1376-88

3. Jayson GC, Kerbel R, Ellis LM, Harris AL. Antiangiogenic therapy in oncology: current status and future directions. Lancet. 2016;388(10043):518-29.

4. Burger RA, Brady MF, Bookman MA, Fleming GF, Monk BJ, Huang H, et al. Incorporation of bevacizumab in the primary treatment of ovarian cancer. N Engl J Med. 2011;365(26):2473-83.

5. Perren TJ, Swart AM, Pfisterer J, Ledermann JA, Pujade-Lauraine E, Kristensen $\mathrm{G}$, et al. A phase 3 trial of bevacizumab in ovarian cancer. N Engl J Med. 2011;365(26):2484-96.

6. Oza AM, Cook AD, Pfisterer J, Embleton A, Ledermann JA, Pujade-Lauraine E, et al. Standard chemotherapy with or without bevacizumab for women with newly diagnosed ovarian cancer (ICON7): overall survival results of a phase 3 randomised trial. Lancet Oncol. 2015;16(8):928-36.

7. Tewari KS, Burger RA, Enserro D, Norquist BM, Swisher EM, Brady MF, et al. Final overall survival of a randomized trial of bevacizumab for primary treatment of ovarian cancer. J Clin Oncol. 2019:37(26):2317-28.

8. Backen A, Renehan AG, Clamp AR, Berzuini C, Zhou C, Oza A, et al. The combination of circulating Ang1 and Tie2 levels predicts progression-free survival advantage in bevacizumab-treated patients with ovarian cancer. Clin Cancer Res. 2014;20(17):4549-58.

9. Zhou C, Clamp A, Backen A, Berzuini C, Renehan A, Banks RE, et al. Systematic analysis of circulating soluble angiogenesis-associated proteins in ICON7 identifies Tie2 as a biomarker of vascular progression on bevacizumab. Br J Cancer. 2016;115(2):228-35.

10. Jayson GC, Zhou C, Backen A, Horsley L, Marti-Marti K, Shaw D, et al. Plasma Tie2 is a tumor vascular response biomarker for VEGF inhibitors in metastatic colorectal cancer. Nat Commun. 2018;9(1):4672.

11. Bergers $G$, Hanahan D. Modes of resistance to anti-angiogenic therapy. Nat Rev Cancer. 2008:8(8):592-603.

12. Lu KV, Chang JP, Parachoniak CA, Pandika MM, Aghi MK, Meyronet D, et al. VEGF inhibits tumor cell invasion and mesenchymal transition through a MET/NEGFR2 complex. Cancer Cell. 2012;22(1):21-35.

13. Ferrara N, Davis-Smyth T. The biology of vascular endothelial growth factor. Endocr Rev. 1997;18(1):4-25.

14. Trusolino L, Bertotti A, Comoglio PM. MET signalling: principles and functions in development, organ regeneration and cancer. Nat Rev Mol Cell Biol. 2010;11(12):834-48.

15. Lambrechts D, Claes B, Delmar P, Reumers J, Mazzone M, et al. VEGF pathway genetic variants as biomarkers of treatment outcome with bevacizumab: an analysis of data from the AVITA and AVOREN randomised trials. Lancet Oncol. 2012;13(7):724-33.

16. de Haas S, Delmar P, Bansal AT, Moisse M, Miles DW, et al. Genetic variability of VEGF pathway genes in six randomized phase III trials assessing the addition of bevacizumab to standard therapy. Angiogenesis. 2014;17(4):909-20.

17. Gill R, Schumacher M. A simple test of proportional hazards assumption. Biometrika. 1987;74(2):289-300.

18. Therneau TM, Grambsch PM, Fleming TR. Martingale-based residuals for survival models. Biometrika. 1990;77(1):147-60.

19. Altman DG, McShane LM, Sauerbrei W, Taube SE. Reporting Recommendations for Tumor Marker Prognostic Studies (REMARK): explanation and elaboration. PLoS Med. 2012;9(5):e1001216. 
20. R Core Team (2020). R: a language and environment for statistical computing. Vienna: R Foundation for Statistical Computing. (https://www.Rproject.org/)

21. Yakes FM, Chen J, Tan J, Yamaguchi K, Shi Y, Yu P, et al. Cabozantinib (XL184), a novel MET and VEGFR2 inhibitor, simultaneously suppresses metastasis, angiogenesis, and tumor growth. Mol Cancer Ther. 2011;10(12):2298-308

22. Zou HY, Li Q, Lee JH, Arango ME, McDonnell SR, Yamazaki S, et al. An orally available small-molecule inhibitor of c-Met, PF-2341066, exhibits cytoreductive antitumor efficacy through antiproliferative and antiangiogenic mechanisms. Cancer Res. 2007;67(9):4408-17.

23. Choueiri TK, Escudier B, Powles T, Tannir NM, Mainwaring PN, Rini BI, et al. Cabozantinib versus everolimus in advanced renal cell carcinoma (METEOR): final results from a randomised, open-label, phase 3 trial. Lancet Oncol. 2016;17(7):917-27.

24. Elisei R, Schlumberger MJ, Muller SP, Schoffski P, Brose MS, Shah MH, et al. Cabozantinib in progressive medullary thyroid cancer. J Clin Oncol. 2013;31(29):3639-46

25. Solomon BJ, MokT, Kim DW, Wu YL, Nakagawa K, Mekhail T, et al. First-line crizotinib versus chemotherapy in ALK-positive lung cancer. N Engl J Med. 2014;371(23):2167-77.

26. Konstantinopoulos PA, Brady WE, Farley J, Armstrong A, Uyar DS, Gershenson DM. Phase II study of single-agent cabozantinib in patients with recurrent clear cell ovarian, primary peritoneal or fallopian tube cancer (NRG-GY001). Gynecol Oncol. 2018;150(1):9-13.

27. Matulonis UA, Sill MW, Makker V, Mutch DG, Carlson JW, Darus CJ, et al. A randomized phase II study of cabozantinib versus weekly paclitaxel in the treatment of persistent or recurrent epithelial ovarian, fallopian tube or primary peritoneal cancer: an NRG Oncology/Gynecologic Oncology Group study. Gynecol Oncol. 2019;152(3):548-53.

28. Vergote IB, Smith DC, Berger R, Kurzrock R, Vogelzang NJ, Sella A, et al. A phase 2 randomised discontinuation trial of cabozantinib in patients with ovarian carcinoma. Eur J Cancer. 2017:83:229-36.

29. Balikova I, Postelmans L, Pasteels B, Coquelet P, Catherine J, Efendic A, et al. Genetic biomarkers in the VEGF pathway predicting response to anti-VEGF therapy in age-related macular degeneration. BMJ Open Ophthalmol. 2019;4(1):e000273.

30. O'Brien TJ, Harralson AF, Tran T, Gindoff I, Orkunoglu-Suer FE, Frankfurter $D$, et al. Kinase insert domain receptor/vascular endothelial growth factor receptor 2 (KDR) genetic variation is associated with ovarian hyperstimulation syndrome. Reprod Biol Endocrinol. 2014;12:36.

31. Bais C, Mueller B, Brady MF, Mannel RS, Burger RA, Wei W, et al. Tumor microvessel density as a potential predictive marker for bevacizumab benefit: GOG-0218 biomarker analyses. J Natl Cancer Inst. 2017;109(11):djx066.

\section{Publisher's Note}

Springer Nature remains neutral with regard to jurisdictional claims in published maps and institutional affiliations.

Ready to submit your research? Choose BMC and benefit from:

- fast, convenient online submission

- thorough peer review by experienced researchers in your field

- rapid publication on acceptance

- support for research data, including large and complex data types

- gold Open Access which fosters wider collaboration and increased citations

- maximum visibility for your research: over $100 \mathrm{M}$ website views per year

At BMC, research is always in progress.

Learn more biomedcentral.com/submissions 